\title{
In-situ Oxygen Isotopic Exchange Vibrational Spectroscopy of Rhenium Oxide Surface Structures on Cerium Oxide
}

Blake MacQueen ${ }^{\dagger}$, Benjamin Ruiz-Yi ${ }^{\dagger}$, Michael Royko ${ }^{\dagger}$, Andreas Heyden ${ }^{\dagger}$, Yomaira J. Pagan-Torres ${ }^{\ddagger}$, Christopher Williams ${ }^{\dagger}$, Jochen Lauterbach ${ }^{\dagger *}$

+ University of South Carolina, Department of Chemical Engineering, 541 Main St., Columbia, SC, 29208, USA

${ }^{\ddagger}$ Department of Chemical Engineering, University of Puerto Rico-Mayaguez Campus, Mayaguez, Puerto Rico 00681-9000, United States

*Corresponding author: lauteraj@cec.sc.edu

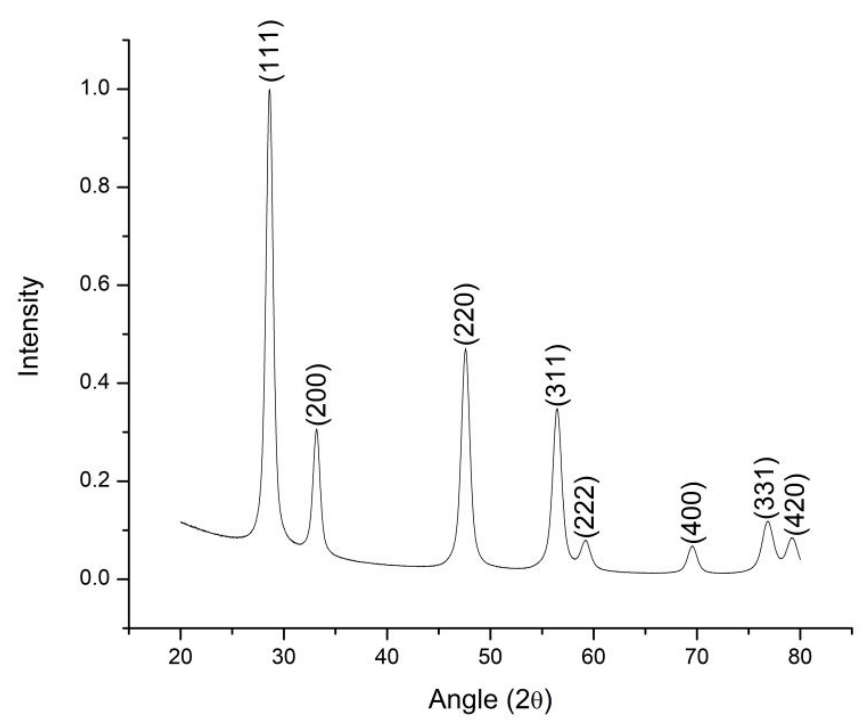

Figure1S. XRD pattern of $1 \mathrm{wt} . \% \mathrm{ReO}_{x} / \mathrm{CeO}_{2}$ at a scanning rate of $0.5^{\circ} \mathrm{min}$. 


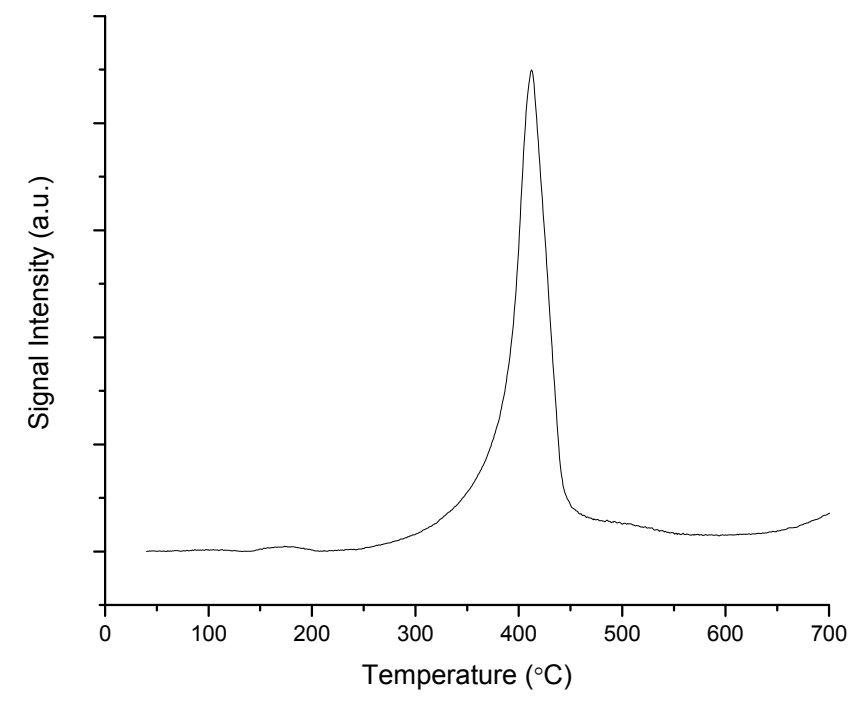

Figure 2S. $\mathrm{H}_{2}$ TPR spectrum of 1 wt. $\% \mathrm{ReO}_{\mathrm{x}} / \mathrm{CeO}_{2}$.

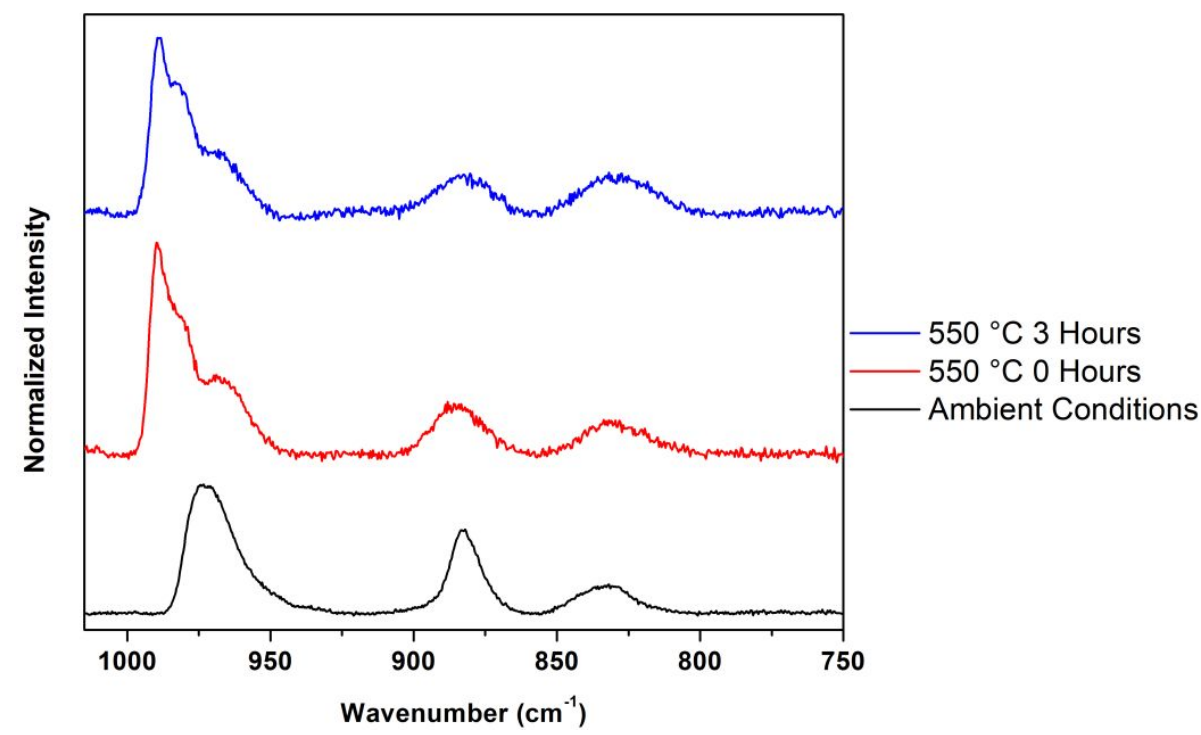

Figure 3S. Raman spectra showing the signal-to-noise comparison between the sample heated at $550{ }^{\circ} \mathrm{C}$ for 0 hours and after 3 hours. 


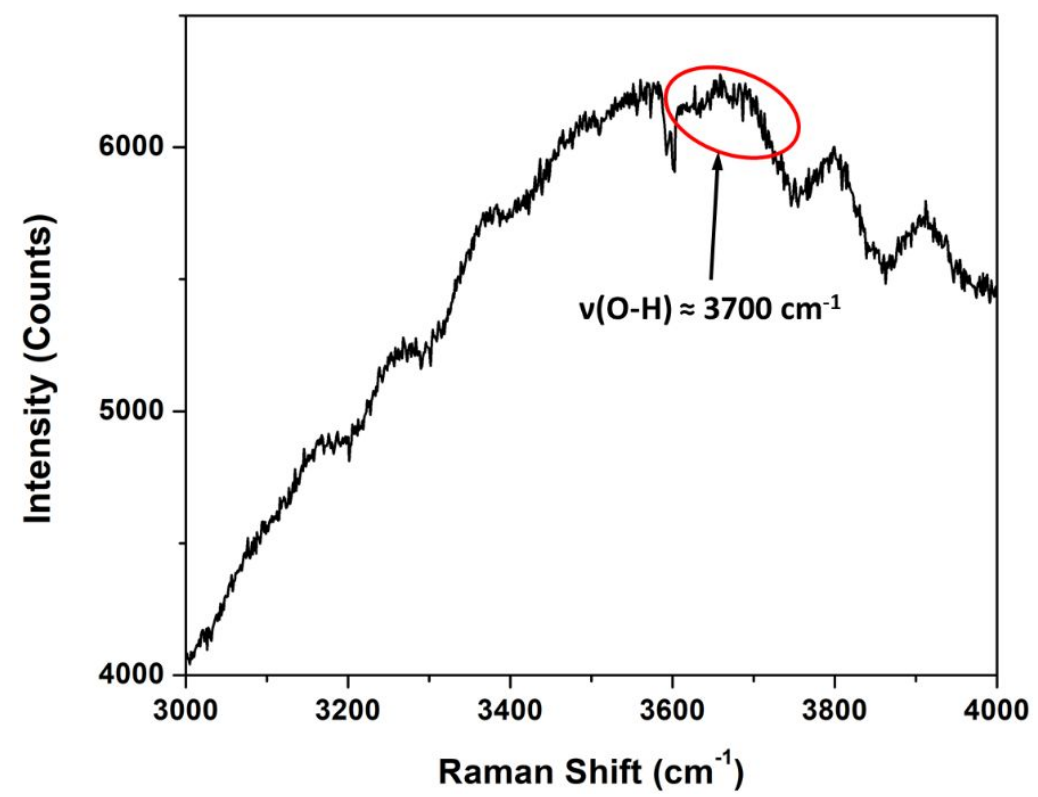

Figure 4S. Raman spectrum of 1 wt.\% ReOx/CeO2 highlighting the O-H stretch.

Table 1S. Raman band area for the observed vibration modes.

\begin{tabular}{|c|c|c|}
\hline \multicolumn{3}{|c|}{ Raman Spectra Area } \\
\hline \multirow{2}{*}{ Vibrational Mode } & \multicolumn{2}{|c|}{$180 / 16$ O Ratio } \\
\cline { 2 - 3 } & ${ }^{18}$ O First Cycle & ${ }^{18}$ O Second Cycle \\
\hline $\mathrm{v}(\operatorname{Re}=0)$ & 1.68 & 9.86 \\
\hline$v_{\text {s }}(\mathrm{O}=\mathrm{Re}=0)$ & 1.37 & 2.45 \\
\hline$v_{\text {as }}(\mathrm{O}=\mathrm{Re}=0)$ & 1.37 & 2.45 \\
\hline$v_{\text {as }}(\operatorname{Re}-\mathrm{O}-\mathrm{Re})$ & 1.09 & Near Complete \\
\hline$\delta(\operatorname{Re}-\mathrm{OH})$ & 1.36 & Near Complete \\
\hline
\end{tabular}


Table 2S. FTIR spectra band area for the visible modes in the spectra after 25 minutes of ${ }^{18} \mathrm{O}$ exposure.

\begin{tabular}{|c|c|c|}
\hline \multicolumn{3}{|c|}{ FTIR Spectra Area } \\
\hline \multirow[b]{2}{*}{ Vibrational Mode } & \multicolumn{2}{|c|}{${ }^{18} \mathrm{O} / 160$ Ratio } \\
\hline & ${ }^{180}$ First Cycle & 180 Second Cycle \\
\hline$v(\operatorname{Re}=0)$ & 0.243 & 1.467 \\
\hline$v_{s}(0=\operatorname{Re}=0)$ & 0.350 & 1.873 \\
\hline $\mathbf{v}_{\mathrm{as}}(\mathrm{O}=\mathrm{Re}=\mathbf{0})$ & 0.350 & 1.873 \\
\hline $\mathbf{v}_{\text {as }}(\mathbf{R e}-\mathbf{O}-\mathbf{R e})$ & 0.148 & 1.298 \\
\hline
\end{tabular}

Table 3S. Ration between the integrated intensities of the symmetric and asymmetric di-oxo stretches in the Raman and FTIR.

\begin{tabular}{|c|c|c|c|}
\hline \multicolumn{4}{|c|}{ Integrated Intensity } \\
\hline & $\mathrm{v}_{\mathrm{s}}(\mathrm{O}=\mathrm{Re}=\mathrm{O})$ & $\mathrm{v}_{\text {as }}(\mathrm{O}=\mathrm{Re}=\mathrm{O})$ & Sym/Asym Ratio \\
\hline Raman & 122.38 & 85.64 & 1.43 \\
\hline Raman (Isotope) & 123.55 & 86.46 & 1.43 \\
\hline FTIR & 0.374 & 0.377 & 0.99 \\
\hline FTIR (Isotope) & 0.662 & 0.669 & 0.99 \\
\hline
\end{tabular}

